\author{
D. McNally \\ University of London Observatory, Mill Hill Park, London NW7 2QS, UK
}

1. Introduction. Classes in Observational Astronomy and Projects can take many forms depending on the type of student for which they have been designed. I wish to consider, in this talk, classes in astronomical observation and individual research projects for final year, first degree, honours students taking the Astronomy (and Astronomy related) degree(s) of University College London. Students in their final year are about to make the transition from university to the work place or into astronomical research. The practical training they receive and projects they undertake must reflect that transition. Since graduates in astronomy will be destined to enter many professions, the only realistic unifying goal must be to prepare the students for astronomical research. To do any less would sell the students short on acquisition of professional experience even though most may never embark on astronomical research.

In earlier years the students will have acquired experience of astronomical observation as well as experience of working in the physics laboratory. They will be acquainted with numerical techniques and be in possession of a considerable range of astronomical information from lecture courses. The aim of the project is to give the student a feeling for astronomical research through choice and execution of an individual project. The philosophy of the final year UCL astronomy project has been discussed at some length in The Role of Projects in Astronomy Education (McNally, 1990) and need not be repeated here. The aim of the observational course is to give the undergraduates a range of experience of observational techniques and data reduction procedures which will give them a good start in astronomical research. There is a considerable element of student choice in selection of experiments to be done but the course contains some compulsory elements. One of these elements is the planning, execution and reporting of an observing programme utilising both a refracting and reflecting telescope. The data obtained from the programmes of observation may be used in other experiments supplemented if necessary (either to extend the dataset or make good the ravages of the weather) with data base material. Considerable emphasis is placed on the reporting of each experiment - the usual course loading is three experiments plus the observational work. The student is expected to indicate how the experiment relates to astronomy, how the data was obtained and how reduced, and to compare their results with similar work in the literature. Attention is paid to tabulation of data and to awareness of sources of error at all stages of data acquisition and reduction. These are stringent demands. The students, for the first time, are in a situation where they are likely to have a limited number of chances to obtain observational data. They will be restricted by the weather and by the visibility of the object to be observed. There may be no opportunity to make a repeat observation in the worst case. The practical work makes cross course demands and requires that initiative be used. Instruction and training in use of telescopes is given as is also the case for data reduction packages. 
The students use the same data reduction packages as are available to the UK astronomical community through STARLINK and are encouraged to approach their own data in a professional way. Instruction in the use of packages is essential as most undergraduate computer studies are not matched to this type of data reduction.

2. The Students. The students in the final year are taking either the Astronomy or Astrophysics or Astronomy and Physics B.Sc. degree. They are familiar with astronomical concepts, basic physics, mathematics and computing. However, it is unwise to presume that knowledge of the theory of CCD detectors, for example, will translate into practice. Indeed it has been found from experience that it is best to start such matters from scratch. Again the students should know how to treat errors, but in practice they may not. Therefore one has to make them aware of the need to adopt good practice and to be aware of random and systematic sources of error. It is also unwise to assume that the student will translate knowledge from lectures on radiative transfer, for example, to interpretation of stellar spectra. The compartmentalisation of course teaching tends to produce students unused to ranging widely over their knowledge and skills in new situations. The students are expected to use their initiative as much as possible. Project supervisors and practical class demonstrators assist when a student is stuck by first assessing the real nature of the problem and directing the students towards finding their own solutions, e.g. by suggesting a data compilation, additional texts and so on. It is an aim to make the students stand on their own feet and act with independence and confidence. It is only through experience that they can learn of the resources available to help them.

3. The Project. The astronomy project in the 3rd (final) year of the Astronomy based degrees at University College London represents a quarter of the workload of that year. The project topic is the choice of each individual student subject only to availability of supervisors, facilities and resources. The project is assessed on progress reports, a final report and a talk. The topic of the project can be on anything that the director of projects considers "astronomical". "Astronomical" is widely interpreted from Earth observation through astrometry, astrophysics, cosmogony, cosmology to history and philosophy of astronomy.

Students have shown a tendency in recent years to choose analytical projects. These are found within the departmental research groups and are based on data already obtained and, in many cases, calibrated and reduced. The student undertakes the final data analysis and attempts some modelling. Therefore student projects have reflected departmental interests in planetary surface studies, analysis of Earth observation, modelling of planetary atmospheres, stellar spectroscopy in the visible and UV, the physics of early type stars. analysis of planetary nebulae, the analysis of $\mathrm{X}$-ray binaries and design studies in astronomical optics. Interest in planetary studies remains high. Relatively few students opt for a project that requires their own observational efforts despite a relaxation of the difficulty of obtaining sufficient observations with the advent of CCD detectors. Those that do make this choice usually do very well and produce a well balanced report - in recent years stellar spectroscopy, the spectroscopy of planetary nebulae and the suitability of CCDs for astrometric work have all been attempted. There are still a few individualists who will go for the esoteric - numerical studies of star formation, numerical stellar dynamics and even the diffusion of Big Bang cosmology into the public conscious- 
ness as derived from newspaper articles and other popular press material. It must be admitted that it is this latter category that gives particular pleasure if only to realise that the rugged, determined astronomical character still lives!

Projects still find favour with staff and students alike. In the case of the students, there remains a wariness of the written report and in particular of the project talk. With the passage of the years, students seem to be coming to terms with the talks though there are still about the same fraction of talk refusers. There is just as much reluctance as ever to state conclusions and defend them even in otherwise highly competent, confident talks. To some extent the project is no longer an innovation but is becoming part of the accepted order of things. The problem now is to persuade students to be adventurous.

4. Observational Astronomy: The Observing Programme. The students are asked to design an observing programme using both $24 " / 18$ " refractor and 24 " reflector. Essentially this has resolved itself as an imaging or spectroscopic activity. The data can be taken with a view to obtaining data for another experiment or it can be for the sake of acquiring images of certain objects. Usually the refractor is used to obtain images for positional measurements (to be used in conjunction with archival images) and the reflector is used to obtain spectra for use in spectroscopic reductions. The student has to plan object availability during the observing season, expected exposure in relation to magnitude, the number of individual images to be obtained, where to place the spectrograph slit etc. The student then bids for observing time at the most appropriate time of year during the practical class. In this way an agreed student priority listing can be established. For example, a student requiring minor planet observations may be restricted to a particular short time slot - in that period this student will have priority. Scheduling is an important aspect to learn and by and large presents little difficulty despite the importunities of climate.

The students are trained in telescope use in an order dictated by the requirements of their observing schedule. They are taught the mode of operation of each telescope and the procedure to be followed in each dome. They are also given instruction in the use of a CCD detector - read out noise, dark current, background, flat fielding and in the system of its control computer. The mode of operation of STARLINK reduction packages are similarly dealt with in special training sessions. It has been found helpful to establish a number of "crib" sheets to give sufficient basic instructions to get started with CCD operations and STARLINK reduction packages. Most students quickly outgrow the "crib" sheets but a few, surprisingly, remain at this level of competence. This underlines an interesting phenomenon that is now appearing - undergraduate antipathy to computers (in contrast to the inability to use computers of a decade ago).

It should be noted that some of the students also need to acquire telescope data for projects and they book time on the telescopes for this purpose. Students with observational projects have a higher priority in the training schedules. All students who require additional observing time than can be provided within the confines of scheduled class times can also book additional observing time. This option is clearly necessary to beat the vagaries of the weather.

5. Observational Astronomy. The experiments all involve reduction, measurement and analysis of observational data. This data may be acquired by the students in their 
observing programmes or be archival observational data obtained previously by former students and/or staff, or may be supplied reduced data or data from a standard data base system. Effectively we have three types of experiments: (a) those based on positional astronomy; (b) those based on photometry; (c) those based on spectroscopy.

The photometry experiments are only one in number - reduction of all sky UBV Photometric Observations. This dearth reflects the poor location of the Observatory for photometric work. However, a pilot experiment on CCD photometry will be instituted using a CCD detector on the 24"/18" Refractor in 1994/95 to establish the viability of relative photometry. It is further hoped to begin IR photometry in the foreseeable future. Positional astronomy runs all the way from determination of the coordinates of a star, through measurements of proper motion to the determination of orbital elements for solar system bodies and binary stars, with the determination of lunar librations thrown in for good measure. The spectroscopic experiments are the best endowed ranging from spectral classification, measurement of radical velocity, gaseous nebulae, unusual stars, determination of mass loss rates for $O B$ stars, Seyfert galaxies, even a study of the Zeeman Effect. In all, the students have 18 experiments, from which to choose three. The choice is not entirely free as certain experiments are mutually exclusive and one experiment has to be chosen from within a specified sublist. Within these constraints, each student can find something to suit their interests and capabilities. Each experiment has a description of the work to be attempted, any additional data required and literature references.

6. Observational Astronomy: Assessment. A standard marking scheme is operated. Each experiment carries a specific number of points. Students must not exceed 80 points total over their choice of three experiments. This is an effort to keep an equitable loading for students. In such a scheme some latitude is allowed since not all experiments carry the same number of points. Additional bonus marks can be awarded for exceptional performance above and beyond the experiment description. The purpose of the bonus marks is to encourage and reward individual initiative.

In general, students perform well in the observational class. The mean performance is at the bottom end of upper second class level. While one might have expected a lower level of performance, it is clear that all students can perform satisfactorily if they give adequate attention to their class work - poor achievement usually correlates with inattention and absenteeism. Excellent performance (as with projects) does not necessarily correlate with other measures of performance in taught classes. Both high flyer and honest plodder can do well (or badly) in the observatory class.

The practical class teaches the student many things: (a) locations of compilations of useful data; (b) what are the really useful textbooks; (c) a range of astronomically useful techniques and data reduction procedures of first resort; (d) a sensitivity to expectation of uncertainty in data; (e) a confidence in ability to tackle a problem; (f) a realisation that critical observations are hard to obtain; and (g) maturity and judgement.

7. Conclusion. Projects and practical classes encourage and promote independence and confidence in the student. In both one can see the tempering of natural enthusiasm with maturity of judgement. Confidence in individual ability also grows. 
Exposure to real data with all its imperfections and shortcomings and the experience of acquiring data through the students' own efforts are very hard lessons initially. Unlike the laboratory where a poor set of measurements may be retrieved and unlike the predigested data sets of textbooks, the students must make something of the data presented to them in the project situation and actually acquire, calibrate and reduce data in a practical class. In the case of the project they must look for sources of error which may weaken any conclusions to be drawn. In the case of observational experiments, they are aware that because of the vagaries of the weather, this chance may be the only chance to get a data set. A few students will be defeated by both situations in a way that they would not be troubled by a laboratory or lecture class. Most students come to terms with the project and observational class and it is one of the pleasures of such teaching to see judgement and confidence grow. Some students develop judgement and confidence to professional levels and it is interesting to note that such students are not necessarily those marked out as able by normal examination assessment procedures. It is this final group that are the most rewarding to teach.

McNally, D. 1990, "The Role of Projects in Astronomy Education" in The Teaching of Astronomy (Eds. Pasachoff and Percy), IAU Colloquium 105, Cambridge University Press, pp. 109-119.

\title{
ASTRONOMY RESEARCH PROJECTS FOR HIGH SCHOOL STUDENTS
}

\author{
John R. Percy
}

Erindale Campus, University of Toronto, Mississauga, Ontario, Canada L5L 1C6

E-mail: percy@astro.utoronto.ca

The excitement of research and discovery is a strong motivation and benefit in both doing and teaching science. The so-called "discovery approach", in which students discover (well-known) scientific concepts for themselves, is an essential aspect of good science teaching. The present paper, however, deals with situations in which high school students carry out original research, and thus derive the extra satisfaction of contributing to scientific knowledge.

This "hands-on" approach to astronomy education is not new. The University of Edinburgh has produced and distributed teaching packages of research-grade materials for many years. The growing education budget of the US National Science Foundation (NSF) has spurred the development of hands-on material in the US in the last decade (Pennypacker 1990).

Some original research projects can be carried out in a classroom setting, and high school teacher Jeff Lockwood (1993) has described his extensive experience in this area. He lists several research-based projects which have been carried out in the US, primarily through NSF funding. These include: CIPE, the Center for Image Processing in Education, and the Image Processing Program, both based at the 